\title{
Equations of State for Poly(vinylidene fluoride)
}

\author{
Hiroshi Tanaka, Koichi Takayama, Toshiyuki OKamoto, \\ and Tetuo TAKEMURA \\ Department of Applied Science, Faculty of Engineering, \\ Kyushu University, Fukuoka 812, Japan
}

(Received April 2, 1982)

\begin{abstract}
Equations of state were determined separately for the crystalline and amorphous parts of Form I and II samples of poly(vinylidene fluoride), assuming a linear relation between the specific volume and the degree of crystallinity. The compressibility data of Form II bulk samples were obtained by dilatometry, and those of the Form I and II crystals were determined by X-ray diffraction at high temperature. Ultrasonic sound velocities of Form I and II crystals were obtained as functions of pressure and temperature, using the logarithmic mixture law for sound velocity. The pressure dependences of the Grüneisen constants $\gamma$ and interchain specific heats $C_{\text {inter }}$ were derived for Form I and II crystals at room temperature. Form I crystals had somewhat larger compressibilities than those of Form II throughout the whole range of temperature investigated, and had a larger $\gamma$ and smaller $C_{\text {inter }}$ at atmospheric pressure, but smaller $\gamma$ at high pressures above $2000 \mathrm{~kg} \mathrm{~cm}^{-2}$. The pressure dependences of $\gamma$ and $C_{\text {inter }}$ were stronger in Form $I$ than in Form II. On the basis of these facts, the difference in behavior of the lattice vibrations of these two crystals under high pressure is discussed.

KEY WORDS Equations of State / Poly(vinylidene fluoride) / Thermal Expansion Coefficient / Compressibility / Grüneisen Constant / Interchain Specific Heat / Lattice Vibration /
\end{abstract}

During the last decade, poly(vinylidene fluoride) (PVDF) has received much attention because of its extraordinary piezoelectric and pyroelectric properties and wide practical use. Recently, the ferroelectric nature of PVDF has been reported by several research groups ${ }^{1-3}$ through observation of the switching current and the P-E hysteresis loop. One very interesting problems that became evident from these observations is the ferroelectric polarization reversal mechanism. At our laboratory, the polarization switching current at high pressure was studied to obtain a basic understanding of this mechanism. ${ }^{4}$ For this purpose, changes in the free volume of the crystalline and amorphous parts with pressure are the most important quantities that had to be evaluated. Furthermore, according to the composite model theory, ${ }^{5} P-V-T$ equations of state for the two parts and the pressure dependence of their dielectric properties should be closely related to piezoelectric and pyroelectric properties under high pressure.

\section{PROCEDURE OF ANALYSIS}

PVDF is a semicrystalline polymer. To estimate the specific volumes of the crystalline and amorphous parts, it is mandatory to use the following linear relation between the specific volumes of a bulk sample $V_{\mathrm{b}}$, the crystalline part $V_{\mathrm{c}}$, and the amorphous part $V_{\text {a }}$ at pressure $P$,

$$
V_{\mathrm{b}}(P) / V_{\mathrm{b} 0}=\chi V_{\mathrm{c}}(P) / V_{\mathrm{c} 0}+(1-\chi) V_{\mathrm{a}}(P) / V_{\mathrm{a} 0}
$$

where $\chi$ is the degree of crystallinity and the subscript zero stands for the corresponding values under atmospheric pressure at a given temperature. $V_{\mathrm{b}}(P)$ and $V_{\mathrm{c}}(P)$ were determined, respectively, by dilatometric and $\mathrm{X}$-ray diffraction experiments under various pressures. The degree of crystallinity was determined by hydrostatic weighing. Thus, the isotherms of the crystailine and amorphous parts were obtained separately from the experimental data on a bulk sample crystallized in Form II, using the relation 


$$
\begin{gathered}
-\left(V_{\mathrm{a}, \text { or cll }}(P)-V_{\mathrm{a} 0 \text {,or cllo }}\right) / V_{\mathrm{a} 0 \text {,or cllo }} \\
=A_{\mathrm{a}, \text { or cll }} P+B_{\mathrm{a}, \text { or cll }} P^{2}+C_{\mathrm{a}, \text { or cll }} P^{3}
\end{gathered}
$$

On the other hand, a bulk sample of Form I was obtained by melt crystallization under high pressure. ${ }^{6} V_{\mathrm{cl}}(P)$ of Form I was determined from its Xray diffraction data.

The Grüneisen constant $\gamma$ in the Mie-Grüneisen equation $^{7} P=P_{\mathrm{p}}+\gamma E_{\mathrm{k}} / V\left(P_{\mathrm{p}}\right.$ is the potential pressure and $E_{\mathrm{k}} / V$ is the vibrational energy per unit volume) represents the contribution of the thermal pressure to the internal pressure of a substance and is a measure of unharmonicity of lattice vibrations. If the Slater equation ${ }^{8} \quad \gamma=-(V / 2)\left(\partial^{2} P / \partial^{2} V\right) /$ $(\partial P / \partial V)-2 / 3$ is available for the present system, the Grüneisen constant can be evaluated by the relation,

$$
\gamma=-B / A^{2}-2 / 3
$$

However, the Slater equation is based on the assumption that the temperature is sufficiently low (nearly equal to $0 \mathrm{~K}$ ) and $\gamma$ is small. In general, the harmonicity of lattice vibrations in crystalline polymers is very large at room temperature. Therefore, eq 3 may not be applicable. Another meth$\operatorname{od}^{9}$ to obtain the Grüneisen constant utilizes the pressure dependence of ultrasonic sound velocity and gives

$$
\gamma=1 / u(\partial u / \partial P)_{T} / \beta_{T}+1 / 3
$$

where $u$ and $\beta_{T}$ are the isothermal sound velocity and compressibility, respectively. To separate the sound velocities $u_{\mathrm{c}}$ and $u_{\mathrm{a}}$ of the crystalline and amorphous parts from the sound velocity $u$ of a bulk PVDF sample, it is necessary to apply the logarithmic law for $u$ to Form II bulk samples of different degrees of crystallinity. The relevant relation is

$$
\log u=\chi \log u_{\mathrm{c}}+(1-\chi) \log u_{\mathrm{a}}
$$

It has shown ${ }^{10}$ that this law holds for polyethylene.

In polymer crystals, the main contribution to the Grüneisen constant comes from the unharmonicity of interchain vibrations. Information about the interchain specific heat, $C_{\text {inter }}$, may therefore be obtained from the Grüneisen constant and the thermal expansion coefficient $\alpha$ using the relation, ${ }^{7}$

$$
C_{\text {inter }}=\alpha V_{\mathrm{c}} / \gamma \beta_{T}
$$

\section{SAMPLE PREPARATION}

Molded discs of a KF polymer kindly supplied from Kureha Chemical Co. were used as the starting samples.

For the ultrasonic experiments, Form II bulk samples of various degrees of crystallinity (30$50 \%$ ) were prepared by heating these samples to $200^{\circ} \mathrm{C}$ and then quenching them to various temperatures in silicone oil. Form II bulk samples used for dilatometric $(49.2 \%)$ and $\mathrm{X}$-ray diffraction measurements $(49.7 \%)$ were prepared by melt crystallization.

Form I bulk samples for X-ray experiments $(51.0 \%)$ were obtained by melt crystallization at $3500 \mathrm{~kg} \mathrm{~cm}^{-2}$. Form I samples for ultrasonic measurements $(51.2 \%)$ were prepared by pressure quenching ${ }^{11}$ in order to prevent the samples from thermal decomposition. Thus, a given sample was melted at $240^{\circ} \mathrm{C}$ under $2000 \mathrm{~kg} \mathrm{~cm}^{-2}$ and then crystallized by increasing the pressure to 4000 $\mathrm{kg} \mathrm{cm}^{-2}$.

\section{EXPERIMENTAL PROCEDURE}

A high pressure X-ray diffraction apparatus equipped with a PSPC probe was used to measure the compression and thermal expansion of Form I and II crystals. We have already reported two types of high pressure X-ray vessels equipped with Be windows. ${ }^{12,13}$ For a simpler setting, a more compact

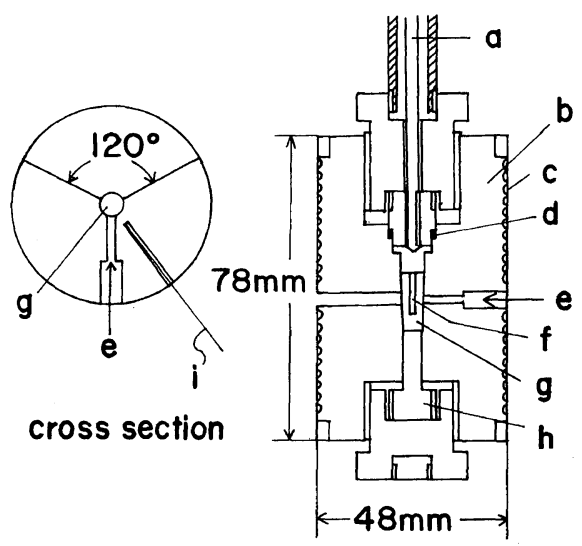

Figure 1. High pressure X-ray diffraction vessel: a, high pressure pipe; $b$, high pressure vessel; $c$, sheathed heater; $d, \mathrm{Cu}$ ring; e, incident beam; f, spacimen; $g$, Be plug; h, supporting plug; i, sheathed thermocouple. 
and handy apparatus was newly constructed (Figure 1). This apparatus allowed us to measure equatorial diffractions at $2 \theta=60^{\circ}$ on both sides of the incident beam (e). The vessel (Nimonic 942) (b) has a Be tapered plug (g) with a small inner hole (1.2 $\mathrm{mm} \phi$ ) for the specimen (f), and its outer diameter and length are $48 \mathrm{~mm}$ and $78 \mathrm{~mm}$, respectively. Its alignment can be completed simply by mounting it on the goniometer head. Pressure is applied through the high pressure pipe (a) from the intensifier. The PSPC system is connected to a minicomputer
(Melcom 80) whose software system can control various experimental conditions, such as the duration of photon counting, and intervals of pressure, temperature, and time between successive data acquisitions. The successive data transferred from the PSPC system to the minicomputer are displayed on a CRT and printed out successively after proper data-processing treatments such as excluding noises from diffraction data, transforming the channel number of the PSPC probe to the diffraction angle, searching the diffraction peak

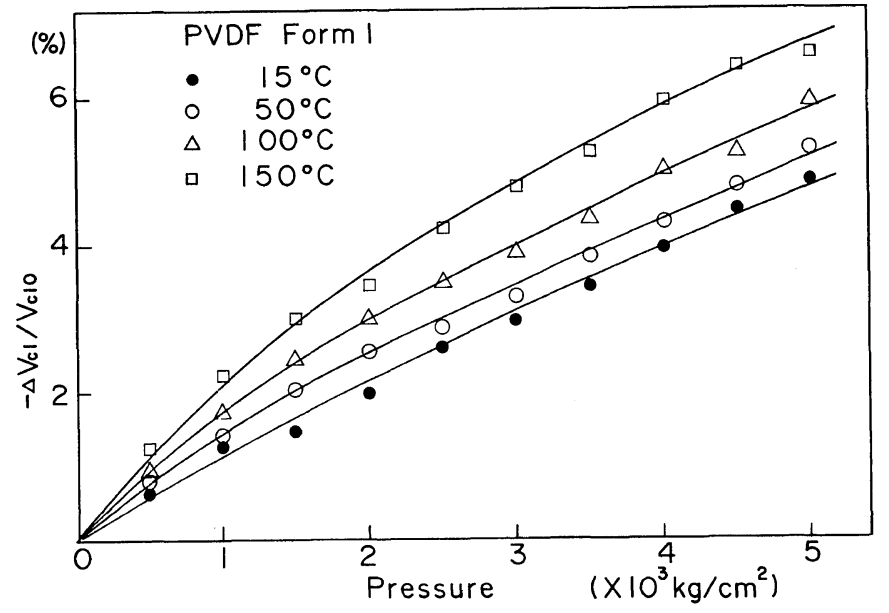

Figure 2. Isothermal pressure-volume relations of a Form I crystal lattice: $O, 15 ; \bigcirc, 50 ; \triangle, 100$; $\square, 150^{\circ} \mathrm{C}$. At each temperature, the volume change is normalized with respect to that atmospheric pressure.

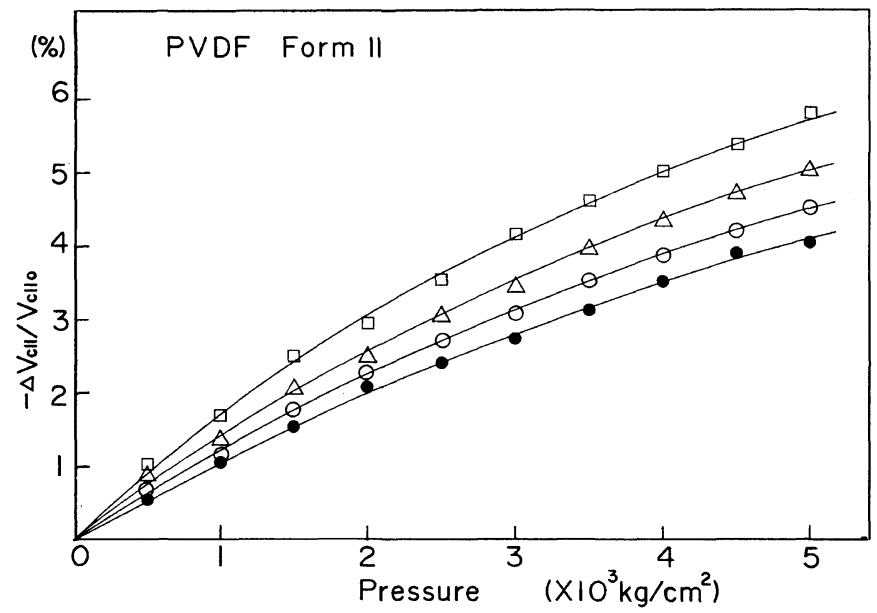

Figure 3. Isothermal pressure-volume relations of a Form II crystal lattice. Symbols are the same as in Figure 2. 


\section{H. TANAKA et al.}

angles (lattice constant or lattice strains), and, if necessary, subtracting the base line associated with the Be window.

The lattice compressive strains of a Form II crystal were obtained from the (110), (020), and (100) reflections. $V_{\text {cII }}$ was determined from these data. Those of a Form I crystal were obtained from the overlapped $(200,110)$ reflection, and $V_{\mathrm{cI}}$ was

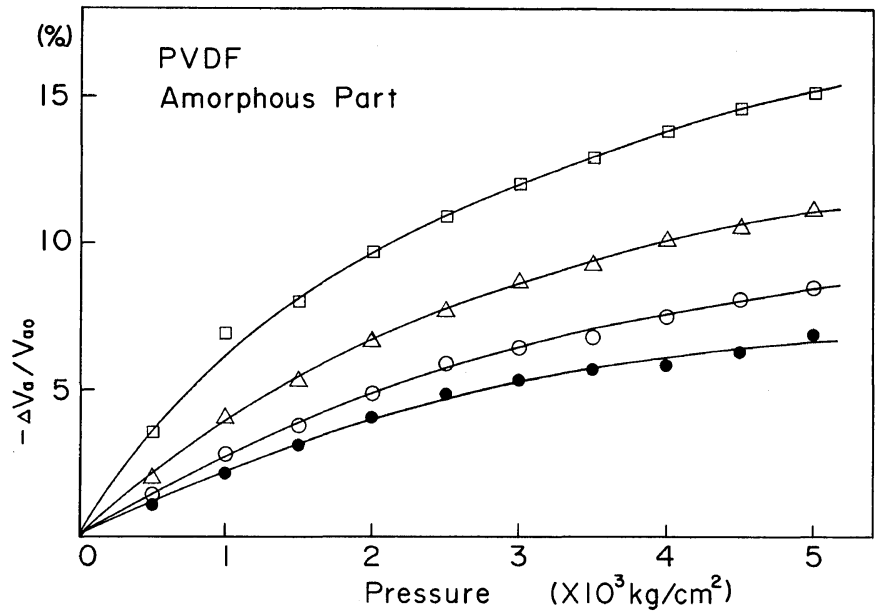

Figure 4. Isothermal pressure-volume relations of amorphous part. Symbols are the same as in Figure 2.

Table I. Best fitting curves for isothermal pressure-strain $(\varepsilon)$ and pressure-volume relations of Form I and II crystal lattices

\begin{tabular}{|c|c|c|c|c|c|}
\hline \multicolumn{3}{|c|}{$-\Delta V / V_{0}(\varepsilon)=A P+B P^{2}+C P^{3}$} & \multicolumn{2}{|c|}{ (Pressure unit; $10^{3} \mathrm{~kg} \mathrm{~cm}^{-2}$ ) } & \multirow[b]{2}{*}{$150^{\circ} \mathrm{C}$} \\
\hline \multicolumn{2}{|c|}{ Temperature } & $15^{\circ} \mathrm{C}$ & $50^{\circ} \mathrm{C}$ & $100^{\circ} \mathrm{C}$ & \\
\hline Form II & $A \times 10^{3}$ & 5.93 & 6.20 & 7.89 & 9.95 \\
\hline \multirow{2}{*}{$\varepsilon_{100}$} & $B \times 10^{4}$ & -5.01 & -3.38 & -8.56 & -11.19 \\
\hline & $C \times 10^{5}$ & 2.86 & 2.17 & 6.20 & 6.51 \\
\hline Form II & $A \times 10^{3}$ & 6.04 & 7.22 & 7.57 & 8.88 \\
\hline \multirow{2}{*}{$\varepsilon_{020}$} & $B \times 10^{4}$ & -4.08 & -8.29 & -6.17 & -7.83 \\
\hline & $C \times 10^{5}$ & 2.29 & 6.01 & 2.43 & 2.54 \\
\hline \multirow{3}{*}{$\begin{array}{l}\text { Form I } \\
\qquad \varepsilon_{200,110}\end{array}$} & $A \times 10^{3}$ & 6.21 & 8.59 & 10.38 & 12.37 \\
\hline & $B \times 10^{4}$ & -6.09 & -1.45 & -17.95 & -18.65 \\
\hline & $C \times 10^{5}$ & 7.43 & 1.64 & 18.60 & 15.36 \\
\hline \multirow[t]{3}{*}{$-\Delta V_{\text {CII }} / V_{\text {CuI }}$} & $A \times 10^{3}$ & 11.97 & 13.42 & 15.46 & 18.84 \\
\hline & $B \times 10^{4}$ & -9.81 & -11.67 & -14.73 & -19.02 \\
\hline & $C \times 10^{5}$ & 5.16 & 6.23 & 8.63 & 9.05 \\
\hline \multirow{3}{*}{$-\Delta V_{\mathrm{Cl}} / V_{\mathrm{CIO}}$} & $A \times 10^{3}$ & 12.42 & 17.18 & 20.76 & 24.74 \\
\hline & $B \times 10^{4}$ & -12.17 & -28.98 & -35.90 & -37.30 \\
\hline & $C \times 10^{5}$ & 14.87 & 32.78 & 37.20 & 30.72 \\
\hline \multirow[t]{3}{*}{$-\Delta V_{\mathrm{a}} / V_{\mathrm{a} 0}$} & $A \times 10^{3}$ & 27.25 & 32.56 & 45.50 & 73.41 \\
\hline & $B \times 10^{4}$ & -41.68 & -46.58 & -69.55 & -147.1 \\
\hline & $C \times 10^{5}$ & 27.76 & 30.92 & 46.38 & 123.1 \\
\hline
\end{tabular}


calculated assuming that the compressive strains along the $a$ - and $b$-axes are equal. In both cases, the strain along the $c$-axis was neglected. Measurement of the thermal expansion of Form I and II crystal lattices was carried out by the same method.

A differential transformer type dilatometer reported previously ${ }^{14}$ was used for the measurement of $V_{\mathrm{b}}$.

Measurement of sound velocities $u$ was performed with a rapid measurement system provided with the Time-to-Amplitude Converter (TAC) system reported previously. ${ }^{10}$ The r.f. pulse buffers method $(5 \mathrm{MHz})$ is suitable for polymers under high pressure.

\section{RESULTS}

1. Compressibilities and Thermal Expansion Coefficients

Figures 2 and 3 show the isothermal $P$ vs.

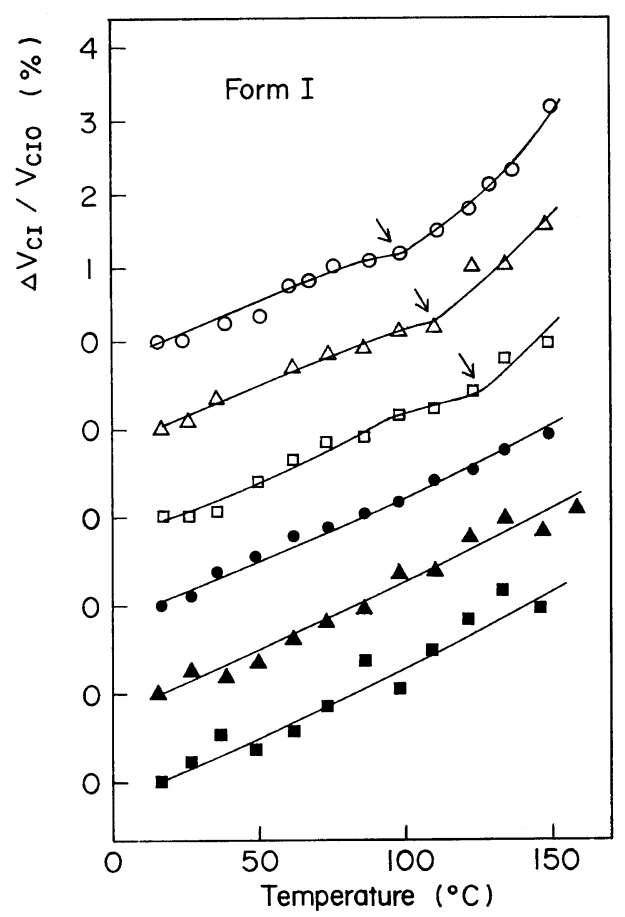

Figure 5. Isobaric temperature-volume curves of a Form I crystal lattice. At each pressure, the volume change is normalized with respect to that at room temperature: $O$, atmospheric pressure; $\triangle, 1000 ; \square$, 2000; , 3000; $\Delta, 4000 ; \square, 5000 \mathrm{~kg} \mathrm{~cm}^{-1}$. The curves, except at atmospheric pressure, are shifted along the vertical axis. The arrows denote the $\alpha$ relaxation points.
$\Delta V_{\mathrm{c}} / V_{\mathrm{c} 0}$ relations for Form I and II crystal lattices determined by $\mathrm{X}$-ray diffraction, respectively. Figure 4 shows the $P$ vs. $\Delta V_{\mathrm{a}} / V_{\mathrm{a} 0}$ relations derived by eq 1 and the dilatometric results for a Form II bulk sample $(49.2 \%$ degree of crystallinity). The best fits of eq 2 to the $P$ vs. $\varepsilon$ ( $\varepsilon$ is the lattice compressive strain) and $P v s . \Delta V / V_{0}$ relations were achieved by the least-squares method; the coefficients $A, B$, and $C$ are listed in Table I.

The thermal expansions of Form I and II crystal lattices at high pressure were measured up to $150^{\circ} \mathrm{C}$ under isobaric conditions. The results are shown in Figures 5 and 6 . The arrows in these figures denote the $\alpha$ relaxation points.

\section{Sound Velocities and the Grüneisen Constants}

The sound velocities of Form II bulk samples of various degrees of crystallinity were measured under isothermal conditions, and eq 5 was applied to determine the pressure dependence of $u_{\mathrm{cII}}$ and $u_{\mathrm{a}}$. The results are shown in Figures 7 and 8, respectively. The pressure dependence of $u_{\mathrm{cI}}$ derived

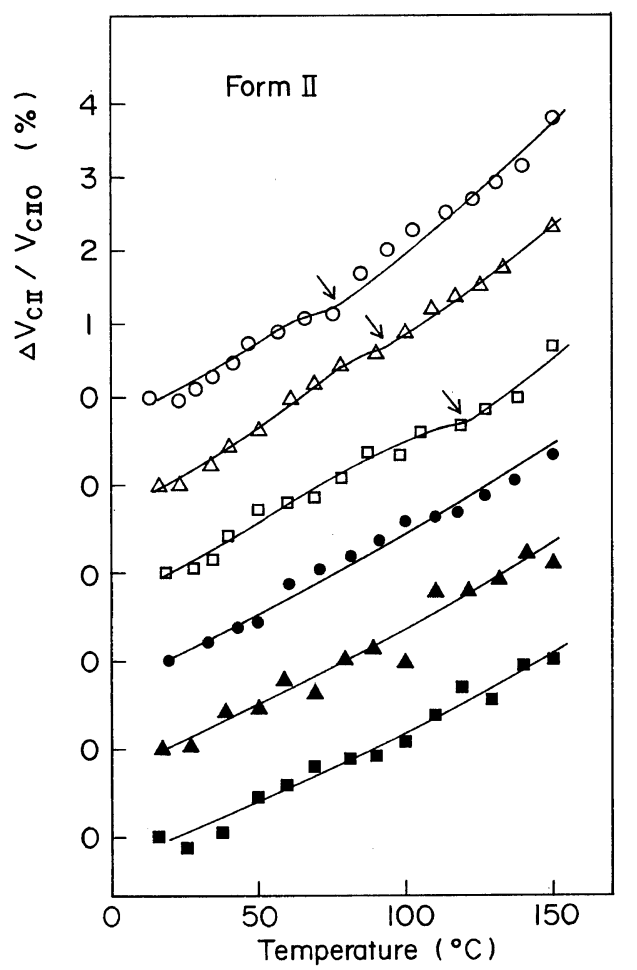

Figure 6. Isobaric temperature-volume relations of a Form II crystal lattice. Symbols are the same as in Figure 5. The arrows denote the $\alpha$ relaxation points. 
H. TANAKA et al.

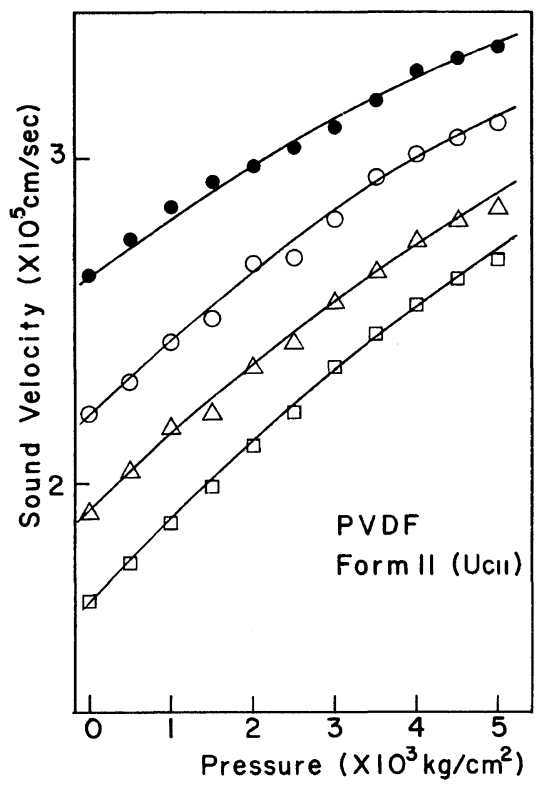

Figure 7. Isothermal pressure-sound velocity curves of a Form II crystal lattice. Symbols are the same as in Figure 2.

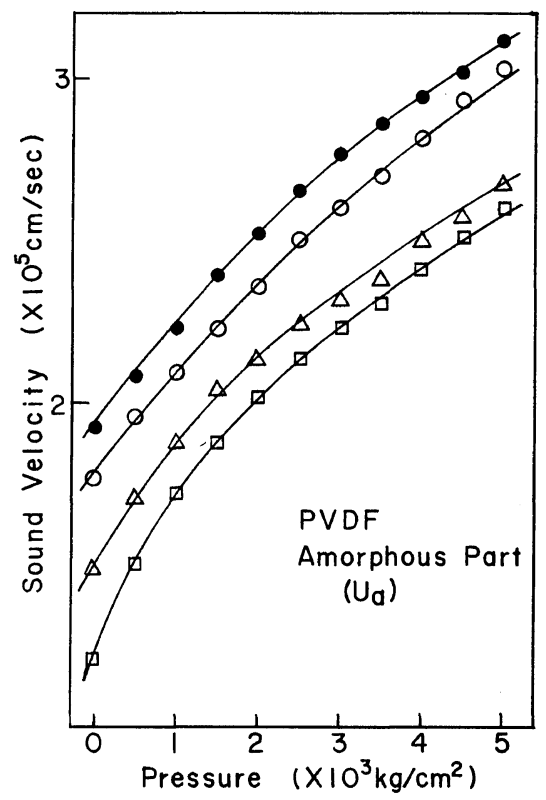

Figure 8. Isothermal pressure-sound velocity curves of amorphous part. Symbols are the same as in Figure 2.

from the values of $u_{\mathrm{a}}$ obtained above and those of $u$ measured on a Form I bulk sample $(51.2 \%$ degree of crystallinity) is illustrated in Figure 9. It can be seen

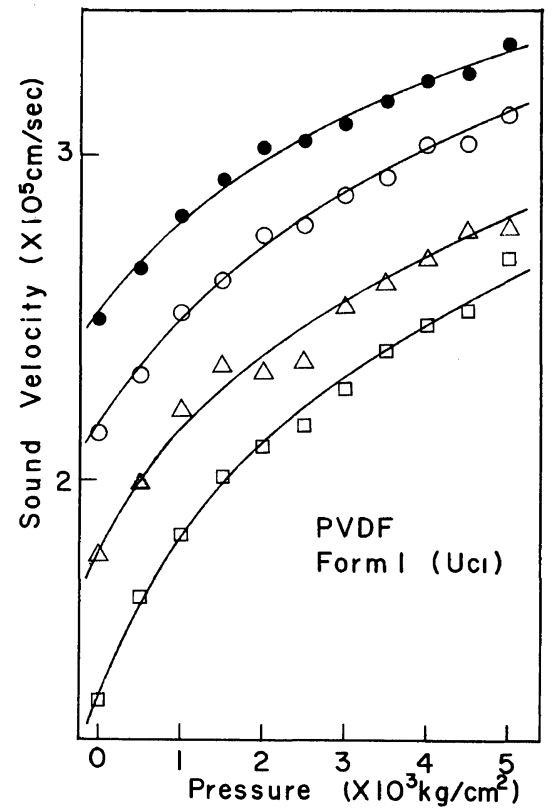

Figure 9. Isothermal pressure-sound velocity curves of a Form I crystal lattice. Symbols are the same as in Figure 2.

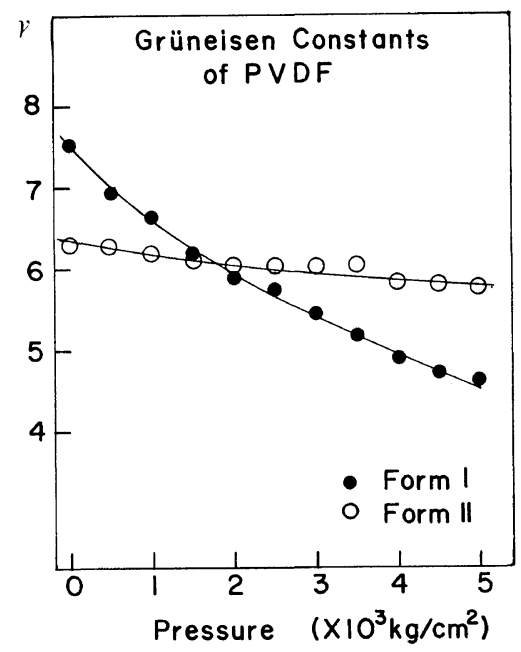

Figure 10. Pressure dependence of the Grüneisen constants of Form I and II crystal lattices at $15^{\circ} \mathrm{C}$. , Form I; O, Form II.

that the sound velocity of the amorphous part has the greatest pressure dependence.

According to eq 4, the Grüneisen constants of Form I and II crystals may be calculated from the above mentioned data and the compressibility data. 


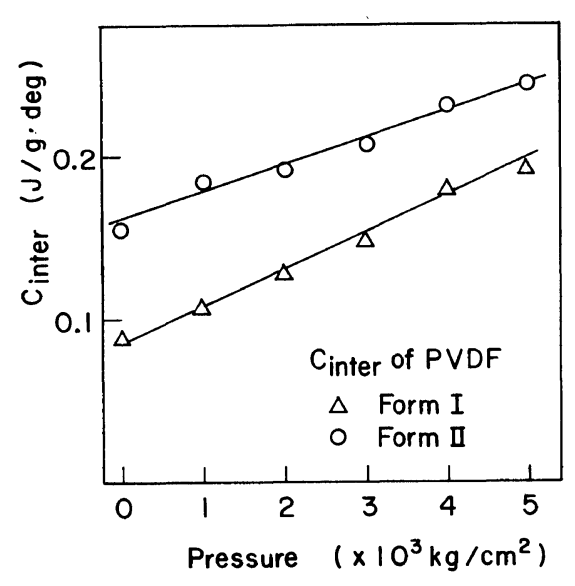

Figure 11. Pressure dependence of the interchain specific heat $C_{\text {inter }}$ of Form I and II crystal lattices at $15^{\circ} \mathrm{C} . \triangle$, Form I; O, Form II.

It should be noted here that Figures 7 and 9 show a characteristic difference in pressure dependence between $u_{\mathrm{cl}}$ and $u_{\mathrm{cII}}$. At any temperature investigated, the pressure dependence of $u_{\mathrm{cI}}$ was found to be stronger in the range of low pressure and weaker in the range of high pressure than that of $u_{\text {cII }}$. This difference reflects directly the difference in pressure dependence between $\gamma_{\mathrm{cI}}$ and $\gamma_{\mathrm{cIl}}$. Figure 10 shows the pressure dependences of $\gamma_{\mathrm{cl}}$ and $\gamma_{\mathrm{cII}}$ at $15^{\circ} \mathrm{C}$.

\section{Interchain Specific Heat}

For a detailed discussion of the lattice vibrational properties of Form I and II crystals, the interchain specific heat $C_{\text {inter }}$ was calculated using eq 6 , with the values of 0.507 and $0.520 \mathrm{~cm}^{3} \mathrm{~g}^{-115}$ chosen for the specific volumes of Form I and II crystals at atmospheric pressure, respectively. Figure 11 shows the pressure dependence of $C_{\text {inter }}$ of Form I and II crystal lattices at $15^{\circ} \mathrm{C}$. The $C_{\text {inter }}$ of Form $\mathrm{I}$ is smaller than that of Form II by about 0.06 $\mathrm{Jg}^{-1} \mathrm{deg}^{-1}$ at atmospheric pressure, and exhibits a greater pressure dependence than that of Form II.

\section{DISCUSSION}

\section{Compressibility}

Compressibilities of Form I and II crystal lattices at room temperature were investigated by Ito et al. ${ }^{16}$ and Newman et al. ${ }^{23}$ and their temperature dependence was reported by Nakafuku. ${ }^{24}$ Our compressibility data at $15^{\circ} \mathrm{C}$ are in good agreement with those of Ito et al., ${ }^{16}$ and their temperature dependence of the Form II crystal lattice is quite close to that reported by Nakafuku. ${ }^{24}$ it should be mentioned here that our values for coefficient $C$ in eq 2 include fairly large experimental errors due to the diffuse X-ray patterns of PVDF. An exact determination of this coefficient is not always easy even for polyethylene which has a much more perfect crystal structure than PVDF.

The anisotropy of the linear compressibility of the Form II crystal lattice in the directions of the $a$ and $b$-axes was not recognized throughout the whole range of temperature, as evident from Table I. For the Form I crystal lattice, the anisotropy of compressibility in the $a b$ plane was evaluated theoretically by Tashiro et al. ${ }^{17}$ but was not observed experimentally. ${ }^{16} \mathrm{We}$ are not in a position to discuss this anisotropy, since only the overlapped reflection $(200,110)$ was observed. However, it should be noted that our value $12.42 \times 10^{-6}$ $\mathrm{cm}^{2} \mathrm{~kg}^{-1}$ for the volume compressibility of the Form I crystal lattice at $15^{\circ} \mathrm{C}$ and atmospheric pressure, obtained under the assumption $\varepsilon_{\mathrm{a}}=\varepsilon_{\mathrm{b}}$, is in good agreement with the calculated value $12.48 \times 10^{-6} \mathrm{~cm}^{2} \mathrm{~kg}^{-1}$ of Tashiro et al. ${ }^{17}$

It is apparent from Table I that the Form I crystal lattice shows a somewhat larger volume compressibility than the Form II crystal lattice at any temperature. Kobayashi et al. ${ }^{18}$ calculated the interchain force constants in both Form I and II crystals. According to their results, the average of the force constants in the Form I crystal lattice is smaller than that in the Form II. This is consistent with our results.

The amorphous part shows the largest volume compressibility. For example, when the Form I bulk sample was compressed, the amorphous part initially exhibited compressibilities about two and three times larger than the crystalline part at 15 and $150^{\circ} \mathrm{C}$, respectively. These compressibility data are important for analyzing the switching current behavior under high pressure. Using our compressibility data, Matsushige et al. ${ }^{4}$ showed that the pressure dependence of the polarization switching time can be well explained by the pressure dependence of the free volume in the amorphous part, and suggested that the polarization reversal mechanism is closely related to molecular motions in the amorphous part rather than in the crystalline part. Thus, the polarization reversal begins pref- 
erentially at the boundary between the crystalline and amorphous parts and/or at the defects in the sample.

Furthermore, the piezoelectricity and pyroelectricity of PVDF under high pressure can be reasonably well explained with the aid of these compressibility data, as will be reported soon.

\section{Thermal Expansion Coefficient and Interchain Specific Heat $C_{\text {inter }}$}

Anisotropy in the thermal expansion coefficients in the $a b$ plane was observed for the Form II crystal lattice. The thermal expansion coefficient along the $a$-axis was larger than that along the $b$-axis at any pressure. According to Nakagawa and Ishida ${ }^{19}$ who measured the thermal expansion coefficients of the Form II crystal lattice by the X-ray method at atmospheric pressure, the lattice constants $a$ and $b$ increased by about 1.2 and $0.8 \%$, respectively, in the temperature range from 20 to $120^{\circ} \mathrm{C}$. Our corresponding values were 1.6 and $0.9 \%$, respectively.

As can be seen from Figures 5 and 6, at room temperature, the thermal expansion coefficients of the Form II crystal lattice are somewhat larger at pressures up to $3000 \mathrm{~kg} \mathrm{~cm}^{-2}$ and almost the same at the higher pressures above $4000 \mathrm{~kg} \mathrm{~cm}^{-2}$ as those of the Form I. The thermal expansion coefficient is proportional to $\dot{C}_{\text {inter }}$ (see eq 6). The data for $C_{\text {inter }}$ are shown in Figure 11. The $C_{\text {inter }}$ values of 0.095 and $0.155 \mathrm{~J} \mathrm{~g}^{-1} \mathrm{deg}^{-1}$ were obtained for the Form I and II crystals at atmospheric pressure, respectively. In consideration of the reduced lattice concept proposed by Wada et al., the $C_{\text {inter }}$ in this case may be described by

$$
C_{\text {inter }}=3 R / m(g / f)^{1 / 2}
$$

where $R, m, g$, and $f$ are the gas constant, the molecular weight of the $\mathrm{CF}_{2}-\mathrm{CH}_{2}$ unit in the original lattice, the interchain force constant, and the intrachain force constant, respectively. The $g$ values can be estimated from the compressibility data in Table I to be $8 \times 10^{4}$ and $8.4 \times 10^{4} \mathrm{~kg} \mathrm{~cm}^{-2}$ for Form I and II, respectively. The $f$ values were reported by ${ }^{4}$ Sakurada and $\mathrm{Kaji}^{20}$ to be $181 \times 10^{4}$ and $60 \times 10^{4} \mathrm{~kg} \mathrm{~cm}^{-2}$ for Form I and II, respectively. With these, the values of 0.084 and 0.148 $\mathrm{J} \mathrm{g}^{-1} \mathrm{deg}^{-1}$ were obtained for $C_{\text {inter }}$ of Form I and II crystals, respectively, in good agreement with our experimental results. On the other hand, the $C_{\text {inter }}$ values of Form I and II crystals increased with increasing pressure, which is reasonable because an increase in pressure gives rise to an increase in the interchain interaction owing to a decrease in interchain distance. Further, Figure 11 shows the difference in the lattice vibrational property between Form I and II crystals, which is discussed in the next section.

The $\alpha$ relaxations were observed at about 75 and $100^{\circ} \mathrm{C}$ for Form II and I crystals at atmospheric pressure, respectively (see Figures 5 and 6). The shifts of these relaxation temperatures due to pressure are about 22 and $14^{\circ} \mathrm{C}$ per $1000 \mathrm{~kg} \mathrm{~cm}^{-2}$ for Form II and I, respectively. The shift of $28^{\circ} \mathrm{C}$ per $1000 \mathrm{~kg} \mathrm{~cm}^{-2}$ was reported for Form II by Tabor $e t$ $a l .,^{21}$ which is close to our value. The $\alpha$ relaxation was dilatometrically observed for Form II at $90^{\circ} \mathrm{C}$ at atmospheric pressure, ${ }^{22}$ but no other static experiments have yet been reported on Form I prepared by high pressure crystallization.

\section{Lattice Vibrations of Form I and II Crystal Lattices}

Our results concerning the physical properties of Form I and II crystal lattices at room temperature under high pressure may be summarized as follows. First, the Form I crystal lattice has a slightly larger compressibility than the Form II crystal lattice (Table I). Second, the former has a larger $\gamma$ value at atmospheric pressure and shows a stronger pressure dependence than the latter (Figure 10). Third, the former has a smaller $C_{\text {inter }}$ value and shows a stronger pressure dependence than the latter. From these facts, the difference between the lattice vibrational motions of Form I and II may be interpreted as follows. The reason for the larger $\gamma$ and the smaller $C_{\text {inter }}$ of Form I at atmospheric pressure is that the planar zigzag chain in the Form I crystal lattice is allowed to librate with a fairly large amplitude about the chain axis, and that this librational vibration in Form $I$ is more incoherent than that in Form II. However, at high pressure, such librational vibration of Form I has a more limited amplitude owing to a decrease in the interchain distance, and hence is more harmonic than at atmospheric pressure. This pressure effect on the vibrational property may be stronger in Form I than in Form II, since the compressibility is larger in the former than in the latter, corresponding to the greater pressure dependence of $\gamma$ and $C_{\text {inter }}$ in the former. 


\section{CONCLUSION}

Equations of state were determined separately for Form I and II crystalline parts and the amorphous part of PVDF from compressibility and thermal expansion data obtained by dilatometric and X-ray diffraction measurements at high pressure and high temperature. The compressibility data were used to explore the ferroelectric polarization reversal mechanism in a Form I sample under high pressure. Further, the equations of state are important for investigating the piezoelectricity and pyroelectricity of PVDF at high pressure and temperature.

Ultrasonic sound velocities of Form I and II samples were determined as functions of pressure and temperature, and the pressure dependences of the Grüneisen constant $\gamma$ and the interchain specific heat $C_{\text {inter }}$ in the crystalline parts of these samples at $15^{\circ} \mathrm{C}$ were calculated. Form I had a larger $\gamma$ and a smaller $C_{\text {inter }}$ at atmospheric pressure, but a smaller $\gamma$ at high pressure than Form II. The compressibility and the pressure dependences of $\gamma$ and $C_{\text {inter }}$ were greater in Form I than in Form II. It is suggested from these facts that, at atmospheric pressure, the librational motion of the planar zigzag chain in the Form I crystal lattice has a fairly large amplitude and is more incoherent than the lattice vibration in the Form II crystal lattice. On the other hand, at high pressure, the librational motion is more restricted to have a smaller amplitude as a result of a decrease in the interchain distance and is more harmonic than the lattice vibration in Form II crystals.

Acknowledgements. The authors wish to thank Kureha Chemical Co. for supplying the samples. This work was supported in part by a Grant-in-Aid for Scientific Research from the Ministry of Education, Science and Culture of Japan.

\section{REFERENCES}

1. M. Tamura, K. Ogasawara, N. Ono, and J.
Hagiwara, J. Appl. Phys., 45, 3768 (1974).

2. M. Oshiki and E. Fukada, J. Mater. Sci., 10, 1 (1975).

3. K. Ogasawara, K. Shiratori, and M. Tamura, Rep. Prog. Polym. Phys. Jpn., 19, 313 (1976).

4. K. Matsushige, S. Imada, H. Tanaka, and T. Takemura, Rep. Prog. Polym. Phys. Jpn., 24, 423 (1981).

5. Y. Wada and R. Hayakawa, Jpn. J. Appl. Phys., 15, 2041 (1976).

6. K. Matsushige and T. Takemura, J. Polym. Sci., Polym. Phys. Ed., 16, 921 (1978).

7. U. N. Zharkov and V. A. Kalinin, "Equations of State for Solids at High Pressure," Consultants Bureau, New York, 1971.

8. J. C. Slater, "Introduction to Chemical Physics," McGraw-Hill, New York, 1939.

9. Y. Wada, A. Iani, T. Nishi, and S. Nagai, J. Polym. Sci., $A-2$, 7, 201 (1969).

10. S. Taki, Y. Furuta, and T. Takemura, Rev. Sci. Instrum., 52, 9 (1981).

11. J. Sheinbeim, C. Nakafuku, B. A. Newman, and K. D. Pae, J. Appl. Phys., 50, 4399 (1979).

12. C. Nakafuku and T. Takemura, Jpn. J. Appl. Phys., 14, 599 (1975).

13. M. Yasuniwa, R. Enoshita, and T. Takemura, Jpn. J. Appl. Phys., 15, 1421 (1976).

14. S. Hirakawa and T. Takemura, Jpn. J. Appl. Phys., 7, 814 (1968); ibid., 8, 635 (1969).

15. R. Hasegawa, Y. Takahashi, Y. Chatani, and H. Tadokoro, Polym. J., 3, 600 (1972).

16. T. Ito, S. Fuji, and A. Okazaki, Polym. Prepr. Jpn., 29, 479 (1980).

17. K. Tashiro, M. Kobayashi, H. Tadokoro, and E. Fukada, Macromolecules, 13, 691 (1980).

18. M. Kobayashi, K. Tashiro, and H. Tadokoro, Macromolecules, 8, 158 (1975).

19. K. Nakagawa and Y. Ishida, Kolloid Z.-Z. Polym., 251, 103 (1973).

20. I. Sakurada and K. Kaji, J. Polym. Sci., C, 31, 57 (1970).

21. E. J. Parry and D. Tabor, Polymer, 14, 623 (1973).

22. J. B. Enns and R. Simha, J. Macromol. Sci., Phys., B13, 11 (1977).

23. B. A. Newman, C. H. Yoon, and K. D. Pae, J. Mater. Sci., 14, 2391 (1979).

24. C. Nakafuku, Rep. Prog. Polym. Phys. Jpn., 23, 187 (1980). 